\title{
Effect of a Seamless Device for Instillation on Bladder Instillation of Drug After Transurethral Resection of Bladder Tumor
}

\author{
Liu Qian", Guo Xiaoxia ${ }^{2, *}$ \\ ${ }^{1}$ Department of Nursing, The First Affiliated Hospital of Jinan University, Guangzhou, China \\ ${ }^{2}$ Department of Urology, The First Affiliated Hospital of Jinan University, Guangzhou, China
}

\section{Email address:}

guoxiaoxia2019@sina.com (Guo Xiaoxia)

${ }^{*}$ Corresponding author

\section{To cite this article:}

Liu Qian, Guo Xiaoxia. Effect of a Seamless Device for Instillation on Bladder Instillation of Drug After Transurethral Resection of Bladder Tumor. Journal of Surgery. Vol. 8, No. 2, 2020, pp. 76-80. doi: 10.11648/j.js.20200802.17

Received: March 29, 2020; Accepted: April 16, 2020; Published: April 29, 2020

\begin{abstract}
Objective: We aim to explore the effect of a seamless device for instillation on bladder instillation of drug after transurethral resection of bladder tumor. Methods: There were 24 patients undergoing transurethral resection of bladder tumor in the department of urinary surgery from January 2019 to December 2019, and 150 person-times of bladder instillation of drug in follow-up. We adopted random number table to averagely divide the 150 person-times into experimental group, control group A and control group B. In the experimental group, before bladder instillation of drug, a $50 \mathrm{ml}$ injector was used for dissolution and suction of drug. During instillation of drug, we used heparin cap to connect the injector and catheter to make a seamless device for instillation. In the control group A, before instillation, a $50 \mathrm{ml}$ injector was also used for dissolution and suction of drug, but at the same time, the syringe nozzle was inserted into the horn-shaped catheter orifice to inject the drug. In the control group B, we used a $50 \mathrm{ml}$ injector for dissolution and suction of drug before instillation and then injected to drug into an aseptic bowel. After that, we used a $50 \mathrm{ml}$ medical irrigator to draw in the liquid in the bowl and inserted the irrigator nozzle into the horn-shaped catheter orifice to inject the liquid. We compared operator's satisfaction during instillation, drug leakage and time consumption between the three groups. Results: Operator's satisfaction in the experimental group, control group A and control group B was $100 \%, 12 \%$ and $84 \%$ respectively and there was a significant difference in that between the three groups $\left(\chi^{2}=57.576, \mathrm{P}=0.000\right)$. In terms of drug leakage, there was no leakage in the experimental group, and an average of $11.44 \pm 2.13 \mathrm{ml}$ of leakage in the control group A and an average of $0.77 \pm 1.14 \mathrm{ml}$ of leakage in the control group B. The one-way analysis of variance showed that there was a significant difference in that $(F=1041.089, \mathrm{P}=0.000)$. At last, the time consumption in the experimental group was $9.28 \pm 1.21 \mathrm{~min}$, and the control group A took the longest time $11.58 \pm 1.81 \mathrm{~min}$. The one-way analysis of variance showed that there was a significant difference in time consumption between the three groups $(F=32.947, \mathrm{P}=0.000)$. Conclusions: the self-designed seamless device for instillation in the bladder instillation of drug for patients who underwent transurethral resection of bladder tumor can not only ensure the dosage of drug, avoid iatrogenic exposure but also reduce time consumption and improve medical staff's satisfaction. Hence, the seamless device is worth clinical application.
\end{abstract}

Keywords: Seamless Device for Instillation, Post-transurethral Resection of Bladder Tumor, Bladder Instillation of Drug, Effect

\section{Introduction}

Bladder tumor is one of the tumors of urinary system that are of the highest incidence [1-3]. It commonly occurs in the side walls or posterior wall of bladder and falls into the category of epithelial cell carcinoma [4]. It is reported that $85 \%$ of primary bladder cancer belongs to non-muscle invasive bladder urothelial carcinoma which has been called superficial 
bladder cancer before. Transurethral resection of bladder tumor (TURBT) combined with postoperative bladder instillation of drug is a standard treatment for the non-muscle invasive bladder urothelial carcinoma. However, the tumor residual rate after the first TURBT is up to $33.8-36 \%[5,6]$. Hence, postoperative bladder instillation of drug is an effective way to reduce recurrence rate of bladder tumor and prolong survival of patients with bladder tumor [7]. There is no instillator which can dissolute and draw in drug and connects to the catheter seamlessly so far. Accordingly, we can only use $50 \mathrm{ml}$ injector for dissolution of drug but the nozzle of thee injector cannot connect to the horn-shaped catheter orifice seamlessly, which may cause leakage of the drug liquid. Although a medical irrigator can prevent the leakage, pouring the drug liquid into the irrigator also leads to waste of the drug, iatrogenic exposure, time consumption and other problems. In order to ensure the accurate dosage of the drug, avoid secondary pollution of the drug and medical exposure, and improve operator's satisfaction, we used heparin cap and $50 \mathrm{ml}$ injector to make a seamless device for bladder instillation of drug for patients who needed this treatment and achieved satisfactory results.

\section{Data and Methods}

\subsection{General Data}

We included 24 patients who underwent TURBT in the department of urinary surgery from January 2019 to December 2019 and needed to be treated with bladder instillation of drug after operation. There were 15 males and 9 female, aging from 26 to 86 with an average age of $(60.25 \pm 14.28)$, and there were 150 person-times of bladder instillation of drug in the follow-up. The inclusion criteria were: patients had bladder tumor at the clinical stage of T1N0M0 and bladder instillation of drug was indicated. Instillation of drug was started one week after the operation (excluding the time of acute inflammation of the bladder) once a week for 8 consecutive weeks, and then once a month, until one year after the operation. The exclusion criteria were: (1) patients had combined severe diseases in heart, lung, liver, kidney and other organs; (2) patients had tumor in other organs or tissues. The 150 person-times were randomized into experimental group $(n=50)$, control group $A(n=50)$ and control group $B(n=50)$. In the control group $A$, there were 42 male person-times and 8 female person-times, aging from $45-76$ with an average age of $(63.45 \pm 5.08)$; 38 person-times of single tumor and 12 person-times of multiple tumor; 42 person-times of low-grade tumor and 8 person-times of high-grade tumor; and 31 person-times of bladder instillation of chemotherapeutic drug and 19 person-times of bladder instillation of immunotherapeutic drug. In the control group B, there were 39 male person-times and 11 female person-times, aging from 32-79 with an average age of (61.38 \pm 9.27$) ; 31$ person-times of single tumor and 19 person-times of multiple tumor; 43 person-times of low-grade tumor and 7 person-times of high-grade tumor; and 40 person-times of bladder instillation of chemotherapeutic drug and 10 person-times of bladder instillation of immunotherapeutic drug. In the experimental group, there were 35 male person-times and 15 female person-times, aging from 32-86 with an average age of $(62.23 \pm 8.58) ; 43$ person-times of single tumor and 7 person-times of multiple tumor; 37 person-times of low-grade tumor and 13 person-times of high-grade tumor; and 38 person-times of bladder instillation of chemotherapeutic drug and 12 person-times of bladder instillation of immunotherapeutic drug. There was no significant difference in sex, age, manner of surgery, grade of tumor, drug for instillation and other general information between the three groups $(\mathrm{P}>0.05)$.

\subsection{Manner of Bladder Instillation of Drug}

\subsubsection{Operator and Length of Bladder Instillation}

The bladder instillation of drug for the three groups were performed by two specific trained nurses and doctors. The patients received bladder instillation at the $7^{\text {th }}$ day postoperatively based on their original urinary catheters.

\subsubsection{Bladder Instillation of Drug in the Follow-up After Discharge}

The patient assumed a supine position. Following the sterile urinary catheterization procedure, we inserted an F8 single-lumen latex catheter to drain the urine, and instilled the dissolved drug solution through the horn-shaped mouth of the catheter at the rate of $15-20 \mathrm{ml} / \mathrm{min}$ [8] into the bladder. After that, we flushed the lumen with $3-5 \mathrm{ml}$ of normal saline, clamped the urinary catheter and pulled it out slowly. We then instructed the patients to change positions (left, right, supine, prone) for 15 minutes each, and guided them to discharge the drug solution according to the different retention times of the drug.

In the control group A, a 50ml injector was used to dissolute, draw in and instill $40 \mathrm{ml}$ of drug solution. The operators inserted the nozzle of the $50 \mathrm{ml}$ injector into the horn-shaped mouth of the catheter to inject the drug after finishing draining urine and indwelling the urinary catheter. At the same time, a piece of gauze was placed under the junction of the injector and urinary catheter to absorb the leakage of drug.

In the control group B, a $50 \mathrm{ml}$ injector was used to dissolute and draw in $40 \mathrm{ml}$ of drug solution, and then the $40 \mathrm{ml}$ of drug solution was poured into an aseptic medical bowl. After that, a $50 \mathrm{ml}$ medical irrigator was used to draw in the drug solution in the bowl and its nozzle was inserted into the horn-shaped mouth of the catheter to inject the drug solution. At the same time, a piece of gauze was placed under the junction of the medical irrigator and urinary catheter absorb the leakage of drug.

In the experimental group, a $50 \mathrm{ml}$ injector was used to dissolute, draw in and instill $40 \mathrm{ml}$ of drug solution. After indwelling the urinary catheter, the blind end of the heparin cap was embedded into the horn-shaped mouth of the urinary catheter to make a seamless device for bladder instillation of drug and the needle of the $50 \mathrm{ml}$ injector was inserted into the catheter through the central hole of the heparin cap to inject 
drug. At the same time, a piece of gauze was placed under the junction of the injector and catheter to absorb the leakage of drug.

\subsection{Methods of Evaluation}

\subsubsection{Time Consumption When Using Different Tools for Bladder Instillation of Drug}

The time consumption of dissolving and suctioning the drug, selecting the instilling container, indwelling the urinary catheter, removing the urinary catheter after completing the instillation was calculated to figure out the total time consumption of instillation of the three groups.

\subsubsection{Leakage of Drug When Using Different Tools for Bladder Instillation of Drug}

Micro-scale was used to weigh the gauze pad under the junction between the device for instillation and the urinary catheter before and after instillation. The weight of the drug solution was converted to the amount of the leakage at the ratio of 1: 1 so as to determine the leakage during the instillation. In the control group B, after the instillation was finished, the gauze pad under the junction should be weighed using it to wipe the sterile bowl. The wasted drug during the preparation process plus the leakage during the instillation process were the actual leakage of drug of the control group B.

\subsubsection{Medical Staff's Satisfaction with Different Tools for Bladder Instillation of Drug}

The operators evaluated the convenience, leakproofness and leakage of drug of the devices for instillation after completing the bladder instillation of drug. Their evaluation included "very satisfied", "satisfied", "partly satisfied" and "dissatisfied".

\subsection{Statistical Methods}

We adopted the software SPSS 21.0 to process the data of the current study. the enumeration data were showed by percentage (\%) and tested by $\chi^{2}$ test. The measurement data were presented by mean \pm standard deviation $(\bar{x} \pm s)$ and was analyzed by one-way analysis of variance. The $\mathrm{P}<0.05$ indicated significant difference.

\section{Results}

\subsection{Comparison of Time Consumption Between Different Devices for Instillation}

The total time consumption of the experimental group was lower than that of control group A and B. The one-way analysis of variance showed that there was a significant difference in the total time consumption between the three groups $(\mathrm{F}=32.947, \mathrm{P}=0.000)$ as shown in the Table 1 .

Table 1. Time Consumption of Bladder Instillation of Drug between the Three Groups.

\begin{tabular}{|c|c|c|c|c|c|}
\hline \multirow{2}{*}{ Groups } & \multirow{2}{*}{$\mathbf{N}$} & \multicolumn{4}{|c|}{ Time consumption (min) } \\
\hline & & $8-10$ & $10-12$ & $12-14$ & Average time consumption \\
\hline Control group A & 50 & 9 & 21 & 20 & $11.58 \pm 1.81$ \\
\hline Control group B & 50 & 35 & 14 & 1 & $9.60 \pm 1.51$ \\
\hline Experimental group & 50 & 41 & 9 & 0 & $9.28 \pm 1.21$ \\
\hline$F$ & & & & & 32.947 \\
\hline $\mathrm{P}$ & & & & & 0.000 \\
\hline
\end{tabular}

\subsection{Comparison of Leakage of Drug Between Different Groups}

There was no leakage of drug in the experimental group because a seamless device for instillation was adopted. In the control group A, the average amount of leakage of drug was
$11.44 \pm 2.13 \mathrm{ml}$ and in the control group B, it was $0.77 \pm 1.14 \mathrm{ml}$. The one-way analysis of variance showed that there was a significant difference in the amount of leakage between the three groups $(\mathrm{F}=1041.089, \mathrm{P}=0.000)$ as shown in the Table 2 .

Table 2. Comparison of Leakage of Drug between the Three Groups.

\begin{tabular}{|c|c|c|c|c|c|c|c|}
\hline \multirow{2}{*}{ Groups } & \multirow{2}{*}{$\mathbf{N}$} & \multicolumn{6}{|c|}{ Amount of leakage (ml) } \\
\hline & & $\mathbf{0}$ & $<0.5$ & $0.5-5$ & $5-10$ & $>10$ & Average amount of leakage \\
\hline Control group A & 50 & 0 & 0 & 0 & 6 & 44 & $11.44 \pm 2.13$ \\
\hline Control group B & 50 & 0 & 38 & 12 & 0 & 0 & $0.77 \pm 1.14$ \\
\hline Experimental group & 50 & 50 & 0 & 0 & 0 & 0 & $0 \pm 0.00$ \\
\hline$F$ & & & & & & & 1041.089 \\
\hline $\mathrm{P}$ & & & & & & & 0.000 \\
\hline
\end{tabular}

\subsection{Medical Staff's Satisfaction with Different Devices for Bladder Instillation of Drug Between the Three Groups}

The satisfaction with the device for instillation of the experimental group was higher than that of the control group
$\mathrm{A}$ and $\mathrm{B}$ with a significant difference $\left(\chi^{2}=57.576, \mathrm{P}=0.000\right)$, which suggested that the device for instillation in the experimental group was more convenient to operate than those in the control group A and B as shown in the Table 3. 
Table 3. Operator's Satisfaction with the Device for Instillation.

\begin{tabular}{llllll}
\hline Groups & N & Very satisfied & Satisfied & Partly satisfied & Dissatisfied \\
\hline Control group A & 50 & 0 & 6 & 15 & 29 \\
Control group B & 50 & 18 & 24 & 4 & 4 \\
Experimental group & 50 & 45 & 5 & 0 & 0 \\
$\chi^{2}$ & & 57.576 & & & \\
P & 0.000 & & & \\
\hline
\end{tabular}

\section{Discussion}

\subsection{Self-designed Seamless Device for Bladder Instillation of Drug to Ensure Accurate Dosage of the Drug and Avoid Iatrogenic Exposure}

TURBT is the gold standard treatment for superficial urothelial carcinoma of the bladder currently. Postoperative bladder instillation of drug can reduce the recurrence rate of bladder cancer and prolong the survival of patients with bladder cancer [5, 9-11]. Bladder instillation of drug refers to direct injection of drug into the bladder with the help of a catheter for treatment and prevention. However, at the current stage, there is no special device for dissolution, suction, and instillation together. In the control group A, a $50 \mathrm{ml}$ injector used for dissolving and aspirating the drug is directly inserted into the horn-shaped mouth of the urinary catheter to inject the drug, during which the leakage of the drug solution is serious. To solve this problem, when using disposable catheter with double chambers for instillation of drug, Li Cuijuan et al. connect the injector with the drug solution to the scalp needle, clamp the entrance of the urine bag before injection, and insert the needle into the urinary catheter at the point of $3 \mathrm{~cm}$ below the bifurcation after sterilizing this point [12]. However, because there is a certain angle between the needle and the catheter during puncture, it is easy to pierce the urinary catheter, which increases the risk of leakage and pricking wound. Thus this method is of little value in clinical practice. In the control group $\mathrm{B}$, a medical irrigator is used as a device for instillation to avoid the leakage of drug solution during the instillation process, but after dissolving the drug, the drug solution should be injected into a sterile medical bowl and then sucked with a medical irrigator. There is residual of the drug solution in the bowl, and at the same time, the risk of occupational exposure is also increased during the operation, especially when dissolving BCG, mitomycin, pirarubicin and other high-risk drugs which are commonly used and required occupational protection. Zhang Ying et al. [13] find that the current status of occupational protection of medical staff in the department of urinary surgery when performing bladder instillation is not optimistic, suggesting that we should pay attention to the occupational protection during bladder instillation of drug [14].

In the current study, because the nozzle of the injector cannot be connected to the horn-shaped mouth of the urinary catheter seamlessly, there is leakage to different degrees. The amount of the leakage of the control group A is higher than that of the experimental group and control group B. In the control group B, a medical irrigator is used for instillation of drug, and the nozzle of the irrigator can be connected to the horn-shaped mouth of the urinary catheter seamlessly. Accordingly, there is no leakage during instillation. However, when the drug solution is poured into the sterile bowel, there will be residual of the solution in the bowel. In the experimental group, heparin cap is connected to the catheter seamlessly so as to achieve integration of dissolution, suction and instillation of drug. The blind end of the heparin cap is $0.7 \mathrm{~cm}$ in diameter and the horn-shaped mouth of the catheter is $0.6 \mathrm{~cm}$ in diameter. The material of the urinary catheter is latex and the mouth of it has elasticity. As the horn-shaped mouth of the catheter is inserted into the heparin cap, due to the rebound resilience of the latex, it can be seamlessly connected to the heparin cap. Before instillation, the needle of the injector with drug solution is inserted into the catheter through the central hole and the blind end of the heparin cap to inject the drug into the bladder. As a result, there is no leakage of drug and occupational exposure during instillation.

\subsection{Controllable Time Consumption to Improve Satisfaction with Bladder Instillation of Drug}

In the control group $\mathrm{A}$, the nozzle of a $50 \mathrm{ml}$ injector was inserted into the horn-shaped mouth of the catheter to instill medical solution slowly. The size of the nozzle of the $50 \mathrm{ml}$ injector and the horn-shaped mouth of the urinary catheter do not match. The operator finds that the speed of instillation is proportional to the amount of leakage. In order to reduce the leakage of the solution, the instillation can only be done slowly. The average time of the instillation is $11.58 \pm 1.81 \mathrm{~min}$. The satisfaction of the medical staff on this method is only $12 \%$. In the control group $\mathrm{B}$, the nozzle of the medical irrigator is inserted into the horn-shaped mouth of the urinary catheter to inject the medical solution, and there is no drug leakage during the instillation. However, the involvement of another injecting container is not satisfactory, especially when dissolving BCG, mitomycin, pirarubicin and other high-risk drugs. The overall satisfaction rate is $84 \%$. In the experimental group clinically available heparin cap is used to make a seamless device for bladder instillation of drug, which did not involve any complication during the operation process, and thus is easily accepted by the medical staff. The self-designed device also helps avoid exposure risk. Therefore, the instillation of drug in the experimental group is the most controllable and the time consumption is the lowest among the three groups, and the medical staff's satisfaction with this method is as high as $100 \%$. 


\section{Conclusions}

In summary, due to the long treatment process of bladder instillation of drug for patients with bladder tumors, the particularity of instillation drug, and the lack of a dedicated device for instillation, the medical staff may face the risk of occupational exposure when preparing and instilling the drug, which seriously discourages the medical staff. In the current study, the heparin cap is used to make a seamless device for bladder instillation of drug which can reduce the risk and the time consumption during operation process, and improve the medical staff's satisfaction with the operation process. Therefore, this method is worthy of clinical application and promotion.

\section{References}

[1] Stenzl, A., Cowan, N. C., Santis, M. D., Kuczyk, M. A., Merseburger, A. S., \& Ribal, M. J., et al. (2012). Treatment of muscle-invasive and metastatic bladder cancer: update of the eau guidelines. 62 (2), e45-e46.

[2] Sanchez, A., Wszolek, M. F., Niemierko, A., Clayman, R. H., Drumm, M., \& Rodríguez D, et al. (2018). Incidence, clinicopathological risk factors, management and outcomes of nonmuscle invasive recurrence after complete response to trimodality therapy for muscle invasive bladder cancer. Journal of Urology, 199 (2), 407.

[3] Kaufman, D. S., Shipley, W. U., \& Feldman, A. S. (2009). Bladder cancer. 374 (9685), 239-249.

[4] Claire L. Vale. (2003). Adjuvant chemotherapy in invasive bladder cancer: a systematic review and meta-analysis of individual patient data. European Urology, 361 (9373), 202-206.

[5] Divrik, R. T., Yildirim, Ü. Zorlu, F. \& Özen, H. The Effect of Repeat Transurethral Resection on Recurrence and Progression Rates in Patients With T1 Tumors of the Bladder Who Received Intravesical Mitomycin: A Prospective, Randomized Clinical Trial. The Journal of Urology, 175 (5), 1641-1644.
[6] Sfakianos, J. P., Kim, P. H., Hakimi, A. A., \& Herr, H. W. (2014). The effect of restaging transurethral resection on recurrence and progression rates in patients with nonmuscle invasive bladder cancer treated with intravesical bacillus calmette-guérin. The Journal of Urology, 191 (2), 341-345.

[7] Ursula Elsässer-Beile, Leiber, C., Wetterauer, U., Patrick Bühler, \& Mengs, U. (2005). Adjuvant intravesical treatment with a standardized mistletoe extract to prevent recurrence of superficial urinary bladder cancer. Anticancer research, 25 (6C), 4733-4736.

[8] Yang Shixi, Zhang Xiangzhi. Improvement of Bladder Infusion Chemotherapy and Observation of Its Adverse Reactions. Journal of Yangtze University (Natural Science Edition), 2017, 14 (12): 58-59+62.

[9] Furuse, H., \& Ozono, S. (2010). Transurethral resection of the bladder tumour (turbt) for non-muscle invasive bladder cancer: basic skills. International Journal of Urology, 17 (8), 698-699.

[10] Cao, M., Yang, G., Pan, J., Sun, J., \& Xue, W. (2015). Repeated transurethral resection for non-muscle invasive bladder cancer. International Journal of Clinical and Experimental Medicine, 8 (1), 1416-1419.

[11] Kyung Seok Han, Jae Young Joung, Kang Su Cho, Ho Kyung Seo, Jinsoo Chung, Won Seo Park, Kang Hyun Lee. (2008). Results of repeated transurethral resection for a second opinion in patients referred for nonmuscle invasive bladder cancer: the referral cancer center experience and review of the literature. Journal of Endourology, 22 (12): 2699-2704.

[12] Li Cuijuan, Li Yan, Huang Qiaoyi. Improvement and Nursing Observation of Bladder Infusion Chemotherapy. Chinese Journal of Clinical Rational Drug Use, 2009, 2 (18): 61-62.

[13] Zhang Ying, Liang Hui, Huang Cuiping, Xu Ting, Gao Lian, Ma Xuexia. Investigation of Occupational Protection Status of Urinary Bladder Infusion Chemotherapy. Journal of Nurses Training, 2019, 34 (15): 1419-1422.

[14] Ding, E., Xu, X., \& Jian, X. (2015). Survey of urology nurses on occupation protection from intravesical chemotherapy. Zhonghua Lao Dong Wei Sheng Zhi Ye Bing Za Zhi, 33 (3), 198-200. 